\title{
How do the extinction curves in galaxies evolve?
}

\author{
Ryosuke S. Asano ${ }^{* 11}$, Tsutomu T. Takeuchi ${ }^{1}$, Hiroyuki Hirashita ${ }^{2}$ and Takaya \\ Nozawa $^{3}$ \\ ${ }^{1}$ Department of Particle and Astrophysical Science, Nagoya University, Furo-cho, Chikusa-ku, \\ Nagoya 464-8602, Japan \\ ${ }^{2}$ Institute of Astronomy and Astrophysics, Academia Sinica, P. O. Box 23-141, Taipei 10617, \\ Taiwan \\ ${ }^{3}$ Kavli Institute for the Physics and Mathematics of the Universe (WPI), University of Tokyo, \\ Kashiwa, Chiba 277-8583, Japan \\ E-mail: asano.ryosuke@g.mbox.nagoya-u.ac.jp, \\ takeuchi.tsutomulg.mbox.nagoya-u.ac.jp, \\ hirashita@asiaa.sinica.edu.tw, takaya.nozawa@ipmu.jp
}

We investigate the evolution of extinction curves in galaxies based on our evolution model of grain size distribution. In this model, we considered various processes: dust formation by $\mathrm{SNe}$ II and AGB stars, dust destruction by $\mathrm{SN}$ shocks in the interstellar medium (ISM), metal accretion onto the surface of grains, shattering and coagulation. We find that the extinction curve is flat in the earliest stage of galaxy evolution because the grain size distribution is dominated by large ( $a \gtrsim 0.1 \mu \mathrm{m}$, where $a$ is the grain radius) grains produced by stars. As the galaxy is enriched with dust, shattering becomes effective to produce a large abundance of small grains ( $a \lesssim 0.01 \mu \mathrm{m})$. Then, the total surface area of grains per grain mass becomes large, and grain growth becomes effective at small grain radii, forming a bump at $a \sim 10^{-3}-10^{-2} \mu \mathrm{m}$ on the grain size distribution. Consequently, the extinction curve at ultraviolet (UV) wavelengths becomes steep, and a bump at $1 / \lambda \sim 4.5 \mu \mathrm{m}^{-1}$ ( $\lambda$ : wavelength) on the extinction curve becomes prominent. The galactic age when the extinction curve has the bump is roughly estimated as $t \sim\left(\tau_{\mathrm{SF}} / \mathrm{Gyr}\right)^{1 / 2} \mathrm{Gyr}$, where $\tau_{\mathrm{SF}}$ is the star formation timescale. Once coagulation becomes effective, the extinction curves become flatter, but the UV extinction remains overproduced when compared with the Milky Way extinction curve. This discrepancy can be resolved by introducing a stronger contribution of coagulation. Thus, an interplay between shattering and coagulation could be important to reproduce the Milky Way extinction curve. We conclude that the extinction curves of galaxies change drastically through the galaxy lifetime because the main dust processes that contribute to the grain size distribution change.

The Life Cycle of Dust in the Universe: Observations, Theory, and Laboratory Experiments - LCDU 2013, 18-22 November 2013

Taipei, Taiwan

\footnotetext{
* Speaker.

${ }^{\dagger}$ Fellow of the Japan Society for the Promotion of Science (JSPS)
} 


\section{Introduction}

Dust grains are one of the fundamental ingredients for understanding the formation and evolution of galaxies. In particular, extinction curves, which represent the wavelength dependence of dust extinction, are a powerful tool to explore the physical and optical properties of dust grains (grain size, dust components, and etc.) in galaxies (e.g., [1,2]).

By fitting the extinction curve in the Milky Way (hereafter MW extinction curve), [1] derived the grain size distribution in the Milky Way (hereafter MRN size distribution), $f(a) \mathrm{d} a \propto a^{-3.5} \mathrm{~d} a$ with $a=0.005-0.25 \mu \mathrm{m}$, where $a$ is the grain radius and $f(a) \mathrm{d} a$ is the number density of grains in size interval $[a, a+\mathrm{d} a]$. The MRN size distribution can be realized if the grains are processed by grain-grain collisions (shattering and coagulation). Thus, it is probable that grain-grain collisions are an important process in the Milky Way and perhaps in nearby galaxies in general. In addition, if the metallicity in galaxies is larger than a certain value, the accretion of gas-phase metals on the surface of pre-existing grains (referred to as 'grain growth' in this manuscript) occurs effectively [3]. Since grain growth has the potential to change the grain size distribution (e.g., [4]), the shape of extinction curves may change by grain growth. In fact, the UV slope on extinction curves becomes steeper due to grain growth if the grain size distribution is initially similar to the MRN size distribution.

The various processes affecting the grain size distribution (referred to as 'dust processes' in this manuscript) occur in a way dependent on the metallicity, total dust amount, and grain size distribution, and could be interrelated. Thus, it is mandatory to construct a model by taking into account all dust processes in a unified framework. Recently, [4] have discussed the evolution of the grain size distribution, taking into account all the dust processes based on chemical evolution of galaxies. They showed that the grain size distribution changes drastically with the galactic age because the dominant dust process changes. In view of the discussion in [4], it is expected that the extinction curve also changes with the galactic age due to the change of the dominant dust processes. Therefore, we examine the evolution of extinction curves in galaxies using the dust evolution model developed by [4], and check whether we can reproduce the MW extinction curve.

\section{Model}

We introduce the model constructed by [4]. [4] investigated the evolution of the grain size distribution taking into account the dust formation by SNe II and AGB stars, dust destruction by SN shocks in the interstellar medium (ISM), grain growth in the cold neutral medium (CNM), graingrain collisions (shattering and coagulation) in the warm neutral medium (WNM) and CNM. Grain growth in the WNM was not considered because grain growth is more efficient in dense and cold regions. [4] considered the contribution of the dust processes in the WNM and CNM by introducing the mass fractions of WNM $\left(\sim 6000 \mathrm{~K}, 0.3 \mathrm{~cm}^{-3}\right)$ and $\mathrm{CNM}\left(\sim 100 \mathrm{~K}, 30 \mathrm{~cm}^{-3}\right), \eta_{\mathrm{WNM}}$ and $\eta_{\mathrm{CNM}}$, respectively. The sum of $\eta_{\mathrm{WNM}}$ and $\eta_{\mathrm{CNM}}$ was assumed to be unity. The grain velocities in the two ISM phases derived by [5] were adopted to calculate shattering and coagulation. We assume two dust species, graphite and silicate [4]. For the dust evolution model, the total baryon mass (the sum of the stellar mass and the ISM mass in the galaxy) is constant, and we introduced the star 


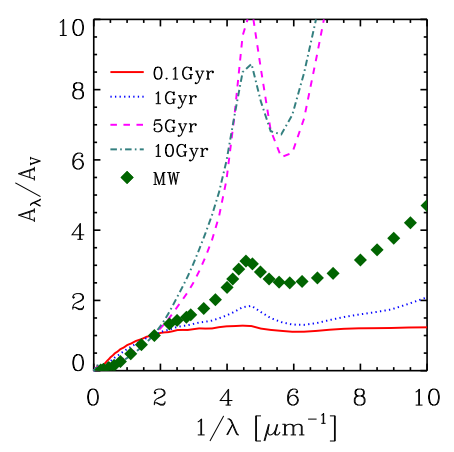

Figure 1: Evolution of the extinction curve. Solid, dotted, dashed and dot-dashed lines represent the cases at $t=0.1,1,5,10 \mathrm{Gyr}$, respectively. Diamonds represent the extinction curve of the Milky Way.
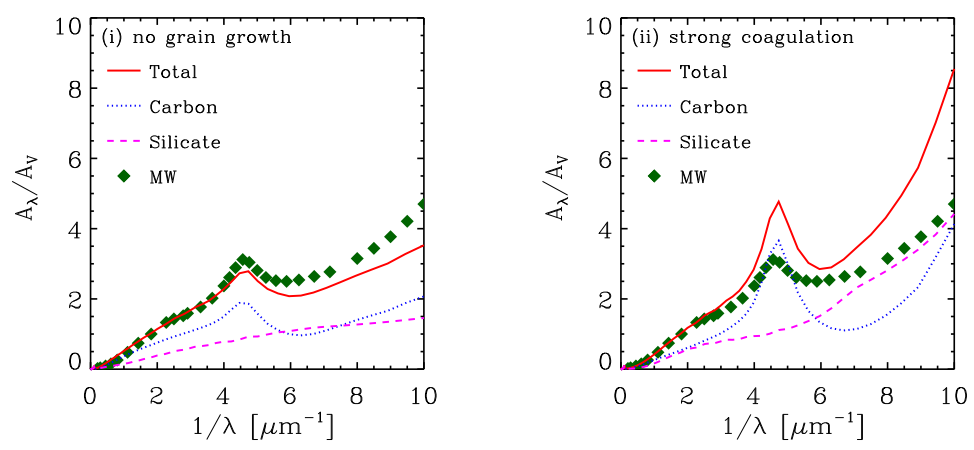

Figure 2: Extinction curves at galactic age $10 \mathrm{Gyr}$ for total grains (solid line), carbon dust (dotted line) and silicate dust (dashed line). Left and right panels show the case (i) without grain growth and (ii) without coagulation threshold velocity. Diamonds represent the extinction curve of the Milky Way.

formation timescale $\tau_{\mathrm{SF}}$ as $\tau_{\mathrm{SF}}=M_{\mathrm{ISM}}(t) / \mathrm{SFR}(t)$, where $M_{\mathrm{ISM}}$ is the ISM mass, SFR is the star formation rate and $t$ is the galaxy age.

\section{Results and Discussion}

Figure 1 shows the evolution of the extinction curve in galaxies taking into account various dust processes. We adopt $\tau_{\mathrm{SF}}=5 \mathrm{Gyr}, \eta_{\mathrm{WNM}}=\eta_{\mathrm{CNM}}=0.5$. We find that, since stellar dust is biased to large grains ( $a \gtrsim 0.1 \mu \mathrm{m}$ ), the extinction curve at the earliest stage of galaxy evolution is flat. After shattering and grain growth occur effectively, the extinction curve becomes steeper and has a larger bump at $1 / \lambda \sim 4.5 \mu \mathrm{m}^{-1}$ than that of the MW extinction curve, where $\lambda$ is the wavelength. After coagulation becomes effective, the bump becomes small.

Next, we examine the possibility of reproducing the MW extinction curve by considering extreme models, (i) no grain growth model and (ii) strong coagulation model. Figure 2 shows the extinction curves for carbon and silicate dust at the galactic age $10 \mathrm{Gyr}$ for the (i) no grain growth model and (ii) strong coagulation model. We adopt $\tau_{\mathrm{SF}}=5 \mathrm{Gyr}, \eta_{\mathrm{WNM}}=\eta_{\mathrm{CNM}}=0.5$. From the panel (i), we find that the calculated extinction curve is similar to that of the Milky Way since the 
shape of the grain size distribution is similar to the MRN size distribution due to the shattering and coagulation. However, if we do not consider the contribution of grain growth to the evolution of the total dust mass in galaxies, it is hard to reproduce the total dust mass of the Milky Way. Thus, by this scenario, we can obtain the extinction curve similar to the MW extinction curve, but has difficulty in reproducing the total dust mass.

For the strong coagulation model [panel (ii)], we observe that the calculated extinction curve is closer to the MW extinction curve than the case in Fig. 1. In this model, since we remove the coagulation threshold velocity ${ }^{1}$, all grains can grow to larger ones by coagulation. Furthermore, thanks to the supply of large grains by strong coagulation, shattering is induced, and as a result, the small grains are supplied. With such an interplay, the size distribution is expected to approach the MRN size distribution. In addition, unlike the model (i), the model (ii) may naturally account for the evolutions of the total dust mass and the grain size distribution and extinction curve in a galaxy at the same time. Thus, we conclude that the strong interplay of shattering and coagulation is important to reproduce extinction curves observed in nearby galaxies.

\section{References}

[1] Mathis J. S., Rumpl W., \& Nordsieck K. H., 1977, The size distribution of interstellar grains, ApJ 217 425

[2] Weingartner J. C., \& Draine B. T., 2001, Dust grain-size distributions and extinction in the Milky Way, Large Magellanic Cloud, and Small Magellanic Cloud, ApJ 548296

[3] Asano R. S., Takeuchi T. T., Hirashita H., \& Inoue A. K., 2013a, Dust formation history of galaxies: a critical role of metallicity for the dust mass growth by accreting materials in the interstellar medium, EPS 65213

[4] Asano R. S., Takeuchi T. T., Hirashita H., \& Nozawa T., 2013b, What determines the grain size distribution in galaxies?, MNRAS $\mathbf{4 3 2} 637$

[5] Yan H., Lazarian A., \& Draine B. T., 2004, Dust dynamics in compressible magnetohydrodynamic turbulence, ApJ 616895

\footnotetext{
${ }^{1}$ Coagulation occurs if the relative velocity of grains is less than the threshold velocity.
} 